OPEN $\curvearrowright$ ACCESS

ISSN 1600-5368

\section{Crystallographic study of PET radio- tracers in clinical evaluation for early diagnosis of Alzheimers ${ }^{1}$}

\author{
Angela Altomare, ${ }^{a}$ Elena Capparelli, ${ }^{\text {b }}$ Antonio Carrieri, ${ }^{\text {b }}$ \\ Nicola A. Colabufo, ${ }^{\mathrm{c}}$ Anna Moliterni, ${ }^{\mathrm{a}}$ Rosanna Rizzi ${ }^{\mathrm{a} *}$ \\ and Dritan Siliqi ${ }^{\mathrm{a}}$
}

a Istituto di Cristallografia, Via G. Amendola, 122/o, 7016, Bari, Italy, bip. di Farmacia-Scienze del Farmaco, Universita' degli studi di Bari, Via Orabona, 4, 70125, Bari, Italy, and ${ }^{\mathbf{c}}$ Dip. di Farmacia-Scienze del Farmaco, Biofordrug, srl, Universita' degli studi di Bari, Via Orabona, 4, 70125, Bari, Italy. *Correspondence e-mail: rosanna.rizzi@ic.cnr.it

Received 3 August 2014; accepted 26 September 2014

Edited by W. T. A. Harrison, University of Aberdeen, Scotland

The title compound, $\mathrm{C}_{24} \mathrm{H}_{25} \mathrm{NO}_{3} \cdot 2 \mathrm{CH}_{3} \mathrm{OH}$, which crystallized as a methanol disolvate, has applications as a PET radiotracer in the early diagnosis of Alzheimer's disease. The dihedral angle between the biphenyl rings is $8.2(2)^{\circ}$ and the heterocyclic ring adopts a half-chair conformation with the $\mathrm{N}$ atom adopting a pyramidal geometry (bond-angle sum = $\left.327.6^{\circ}\right)$. The $\mathrm{C}$ atoms of both methoxy groups lie close to the plane of their attached ring [deviations $=0.107$ (6) and 0.031 (6) $\AA$ ]. In the crystal, the components are linked by $\mathrm{O}-$ $\mathrm{H} \cdots \mathrm{O}$ and $\mathrm{O}-\mathrm{H} \cdots \mathrm{N}$ hydrogen bonds, generating [010] chains. $\mathrm{C}-\mathrm{H} \cdots \mathrm{O}$ interactions are also observed.

Keywords: crystal structure; ligands; P-glycoprotein inhibitor; PET radiotracer; hydrogen bonds.

CCDC reference: 915609

\section{Related literature}

For pharmacological and biological studies of the title compound, see Colabufo et al. (2008, 2009).<smiles>COc1cc2c(cc1OC)CN(Cc1ccc(-c3ccc(O)cc3)cc1)CC2</smiles>

\footnotetext{
$\overline{{ }^{1} \text { Crystal structure }}$ of $4^{\prime}$-[(6,7-dimethoxy-3,4-dihydro- $1 H$-isoquinolin-2-yl)methyl]biphenyl-4-ol methanol disolvate.
}

\section{Experimental}

$\begin{array}{ll}\text { 2.1. Crystal data } & \\ \mathrm{C}_{24} \mathrm{H}_{25} \mathrm{NO}_{3} \cdot 2 \mathrm{CH}_{4} \mathrm{O} & V=1211.8(4) \AA^{3} \\ M_{r}=439.53 & Z=2 \\ \text { Monoclinic, } P_{\circ} & \text { Mo } K \alpha \text { radiation } \\ a=8.894(2) \AA & \mu=0.08 \mathrm{~mm}^{-1} \\ b=13.7187(16) \AA & T=293 \mathrm{~K} \\ c=10.680(2) \AA & 0.30 \times 0.30 \times 0.15 \mathrm{~mm}\end{array}$

\subsection{Data collection}

Bruker-Nonius KappaCCD diffractometer

Absorption correction: multi-scan (SADABS; Sheldrick, 2008a) $T_{\min }=0.921, T_{\max }=0.988$

14813 measured reflections 5436 independent reflections 2610 reflections with $I>2 \sigma(I)$ $R_{\text {int }}=0.116$

2.3. Refinement

$R\left[F^{2}>2 \sigma\left(F^{2}\right)\right]=0.061$

$w R\left(F^{2}\right)=0.115$

$S=0.96$

5436 reflections

305 parameters

1 restraint

$\mathrm{H}$ atoms treated by a mixture of independent and constrained refinement

$\Delta \rho_{\max }=0.17$ e $\AA^{-3}$

$\Delta \rho_{\min }=-0.15$ e $\AA^{-3}$

Table 1

Hydrogen-bond geometry $\left(\AA,^{\circ}\right)$.

\begin{tabular}{lllll}
\hline$D-\mathrm{H} \cdots A$ & $D-\mathrm{H}$ & $\mathrm{H} \cdots A$ & $D \cdots A$ & $D-\mathrm{H} \cdots A$ \\
\hline $\mathrm{O} 2-\mathrm{H} 2 O \cdots \mathrm{N} 1$ & $0.94(5)$ & $1.87(5)$ & $2.812(5)$ & $178(4)$ \\
$\mathrm{O} 4-\mathrm{H} 4 O \cdots \mathrm{O} 5^{\mathrm{i}}$ & $0.95(6)$ & $1.71(6)$ & $2.636(6)$ & $165(5)$ \\
$\mathrm{O} 5-\mathrm{H} 5 O \cdots \mathrm{O} 2$ & $0.71(8)$ & $2.00(8)$ & $2.684(6)$ & $162(9)$ \\
$\mathrm{C} 15-\mathrm{H} 15 A \cdots \mathrm{O} 1^{\mathrm{ii}}$ & 0.97 & 2.50 & $3.445(6)$ & 164 \\
$\mathrm{C} 23-\mathrm{H} 23 A \cdots \mathrm{O} 2^{\mathrm{iii}}$ & 0.96 & 2.56 & $3.437(6)$ & 152 \\
\hline
\end{tabular}

Symmetry codes: (i) $x, y+1, z$; (ii) $-x+1, y+\frac{1}{2},-z$; (iii) $-x, y-\frac{1}{2},-z$.

Data collection: COLLECT (Nonius, 2002); cell determination and refinement: DIRAX (Duisenberg,1992; Duisenberg et al., 2000); data reduction: $E V A L$ (Nonius, 2002; Duisenberg et al., 2003); program(s) used to solve structure: SIR2011 (Burla et al., 2012); program(s) used to refine structure: SHELXL2013 (Sheldrick, 2008b); molecular graphics: ORTEP-3 for Windows (Farrugia, 2012) and EXPO2013 (Altomare et al., 2013); software used to prepare material for publication: WinGX (Farrugia, 2012) and publCIF (Westrip, 2010).

\title{
Acknowledgements
}

This work was supported by the Fondazione Cassa di Risparmio di Puglia (FCRP) of Bari (research project: Studio cristallografico di radiotraccianti PET in valutazione clinica per la diagnosi precoce dell' Alzheimer). The authors thank Mr Giuseppe Chita (Institute of Crystallography CNR, Bari, Italy) for his contribution to the X-ray powder diffraction data collection and Dr Caterina Chiarella (Institute of Crystallography CNR, Bari, Italy) for the technical support to project management. 
Supporting information for this paper is available from the $\mathrm{IUCr}$ electronic archives (Reference: HB7266).

\section{References}

Altomare, A., Cuocci, C., Giacovazzo, C., Moliterni, A., Rizzi, R., Corriero, N. \& Falcicchio, A. (2013). J. Appl. Cryst. 46, 1231-1235.

Burla, M. C., Caliandro, R., Camalli, M., Carrozzini, B., Cascarano, G. L., Giacovazzo, C., Mallamo, M., Mazzone, A., Polidori, G. \& Spagna, R. (2012). J. Appl. Cryst. 45, 357-361.

Colabufo, N. A., Berardi, F., Cantore, M., Perrone, M. G., Contino, M., Inglese, C., Niso, M., Perrone, R., Azzariti, A., Simone, G. M. \& Paradiso, A. (2008). Bioorg. Med. Chem. 16, 3732-3743.
Colabufo, N. A., Berardi, F., Perrone, M. G., Cantore, M., Contino, M., Inglese, C., Niso, M. \& Perrone, R. (2009). ChemMedChem, 4, 188-195.

Duisenberg, A. J. M. (1992). J. Appl. Cryst. 25, 92-96.

Duisenberg, A. J. M., Hooft, R. W. W., Schreurs, A. M. M. \& Kroon, J. (2000) J. Appl. Cryst. 33, 893-898.

Duisenberg, A. J. M., Kroon-Batenburg, L. M. J. \& Schreurs, A. M. M. (2003). J. Appl. Cryst. 36, 220-229.

Farrugia, L. J. (2012). J. Appl. Cryst. 45, 849-854.

Nonius (2002). COLLECT and EVAL. Nonius BV, Delft, The Netherlands.

Sheldrick, G. M. (2008a). SADABS. University of Göttingen, Germany.

Sheldrick, G. M. (2008b). Acta Cryst. A64, 112-122.

Westrip, S. P. (2010). J. Appl. Cryst. 43, 920-925. 


\title{
supporting information
}

Acta Cryst. (2014). E70, o1149-o1150 [doi:10.1107/S1600536814021400]

\section{Crystallographic study of PET radiotracers in clinical evaluation for early diagnosis of Alzheimers}

\author{
Angela Altomare, Elena Capparelli, Antonio Carrieri, Nicola A. Colabufo, Anna Moliterni, \\ Rosanna Rizzi and Dritan Siliqi
}

\section{S1. Comment}

The single-crystal X-ray structure solution of 4'-(6,7-dimethoxy-3,4-dihydro-1 $H$-isoquinolin-2-yl-methyl)-biphenyl-4-ol (named MC70) radiotracer, previously pharmacologically characterized and biologically evaluated (compound 4e in Colabufo et al., 2008, 2009), has been reported. At nanomolar concentrations MC70 is a potent inhibitor of Pglycoprotein (P-gp), a membrane protein playing a protective role of the central nervous system and whose numerical and functional alteration is responsible for the onset of the Alzheimer disease. The crystallographic characterization of MC70 represents the first necessary step for a further evaluation of its pharmacological properties and to obtain, f. e. through docking techniques and homology modelling, a tridimensional interpretation of the main molecular determinants responsible for most of MC70 features such as to be an inhibitor of the P-gp. The study of this behaviour will allow the design of new ligands, more effective and selective in the monitoring the role of P-glycoprotein for the recognition and early treatment of the Alzheimer disease. In addition, up to now none of the studies on interactions of this pump with known inhibitors, report crystallographic data of P-gp inhibitors complexes. Therefore speculating the binding conformation and pose for MC70 might be an added value to a better understanding of the mechanism of action of efflux pumps involved in the Alzheimer's disease.

A view of the refined crystal structure is shown in Figure 1. The packing of the obtained crystal structure is represented in Figure 2; it is interesting observing that the network of the structure features three hydrogen bonds (Table 1): the first between the 2 molecules of methanol, the second between one methanol molecule and the phenolic residue of the molecule and the last between the other methanol molecule and the isoquinoline nucleus. In the crystal weak $\mathrm{C}-\mathrm{H} \cdots \mathrm{O}$ hydrogen bonds also occur. In addition, the pendant biphenyl has an equatorial configuration as proved by a dihedral angle among atoms $\mathrm{C} 8-\mathrm{N} 1-\mathrm{C} 7-\mathrm{C} 18$ of $-175^{\circ}$.

\section{S2. Experimental}

MC70, [4'-(6,7-dimethoxy-3,4-dihydro-1 $H$-isoquinolin-2-yl-methyl)-biphenyl-4-ol] $\left(\mathrm{C}_{24} \mathrm{H}_{25} \mathrm{NO}_{3}\right)$ has been obtained after crystallization as yellow needles. The solvent/non-solvent diffusion has been used as crystallization technique: after solubilizing MC70 (5 mg) in methanol (solvent, $1 \mathrm{ml}$ ), an equal volume of $\mathrm{CH}_{2} \mathrm{Cl}_{2}$ (non-solvent, $1 \mathrm{ml}$ ) has been deposited. The vial has been covered with a perforated cap and left at room temperature. After a couple of days, yellow needles of $\mathrm{MC} 702 \mathrm{CH}_{3} \mathrm{OH}$ have grown on the interphase solvent/non-solvent.

\section{S3. Refinement}

The hydrogen atoms of the hydroxyl groups were located by difference Fourier synthesis and freely isotropically refined. The C-bonded $\mathrm{H}$ atoms were positioned geometrically with $\mathrm{C}-\mathrm{H}=0.96,0.97$ and $0.93 \AA$ for methyl, methylene and 
aromatic $\mathrm{H}$ atoms, respectively, and constrained to ride on their parent atoms. The constraint $U_{\mathrm{iso}}(\mathrm{H})=k U_{\mathrm{eq}}(\mathrm{C})$, where $k=$ 1.5 for methyl and $k=1.2$ for aromatic and methylene $\mathrm{H}$ atoms, was applied. The highest residual electron density was found $1.59 \AA$ from $\mathrm{C} 16$ and the deepest hole $1.04 \AA$ from $\mathrm{H} 12 \mathrm{~A}$.

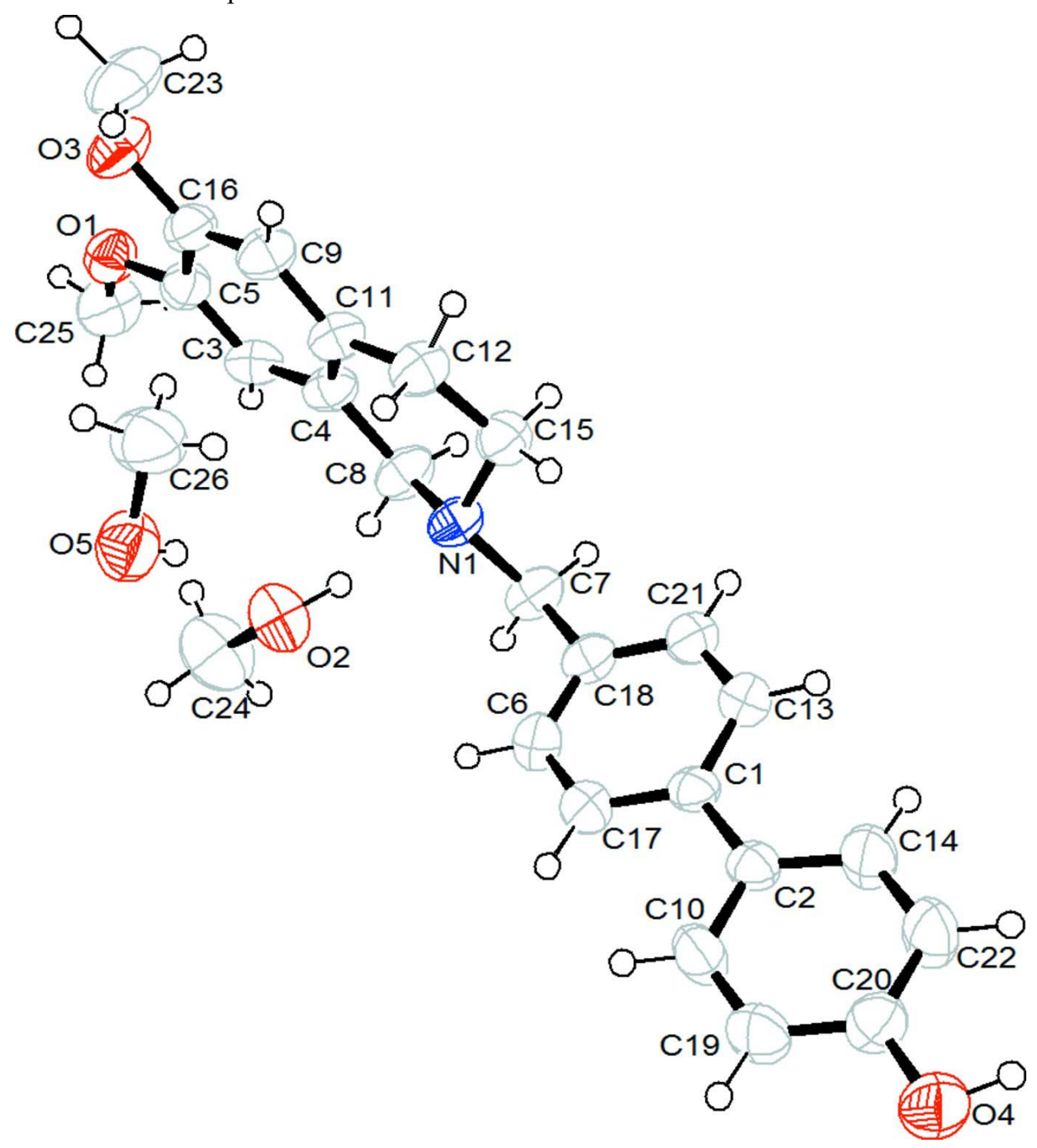

\section{Figure 1}

The molecular structure of the MC70 compound with displacement ellipsoids drawn at the $50 \%$ probability level. 


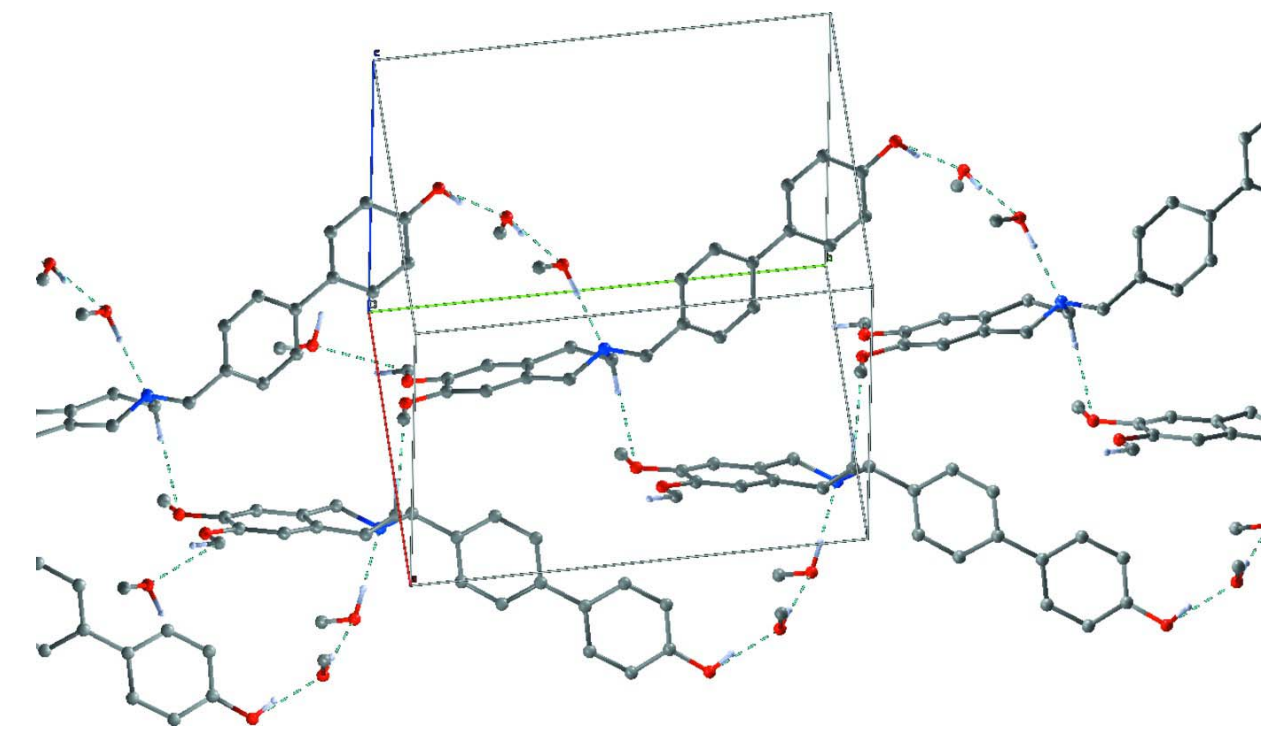

Figure 2

Crystal packing of the MC70 compound. The light blue dashed lines show the hydrogen bonds (see Table 1 for details).

\section{4'-(6,7-Dimethoxy-3,4-dihydro-1 $H$-isoquinolin-2-yl-methyl)-biphenyl-4-ol methanol disolvate}

Crystal data

$\mathrm{C}_{24} \mathrm{H}_{25} \mathrm{NO}_{3} \cdot 2 \mathrm{CH}_{4} \mathrm{O}$

$M_{r}=439.53$

Monoclinic, $P 2_{1}$

$a=8.894(2) \AA$

$b=13.7187(16) \AA$

$c=10.680(2) \AA$

$\beta=111.575(17)^{\circ}$

$V=1211.8(4) \AA^{3}$

$Z=2$

\section{Data collection}

Bruker-Nonius KappaCCD diffractometer

Radiation source: fine-focus sealed tube

Detector resolution: 9.091 pixels $\mathrm{mm}^{-1}$

$\varphi$ scans and $\omega$ scans

Absorption correction: multi-scan

(SADABS; Sheldrick, 2008a)

$T_{\text {min }}=0.921, T_{\text {max }}=0.988$

\section{Refinement}

Refinement on $F^{2}$

Least-squares matrix: full

$R\left[F^{2}>2 \sigma\left(F^{2}\right)\right]=0.061$

$w R\left(F^{2}\right)=0.115$

$S=0.96$

5436 reflections

305 parameters

1 restraint
$F(000)=472$

$D_{\mathrm{x}}=1.205 \mathrm{Mg} \mathrm{m}^{-3}$

Mo $K \alpha$ radiation, $\lambda=0.71073 \AA$

Cell parameters from 130 reflections

$\theta=2.9-26.6^{\circ}$

$\mu=0.08 \mathrm{~mm}^{-1}$

$T=293 \mathrm{~K}$

Needle, yellow

$0.30 \times 0.30 \times 0.15 \mathrm{~mm}$

14813 measured reflections

5436 independent reflections

2610 reflections with $I>2 \sigma(I)$

$R_{\text {int }}=0.116$

$\theta_{\text {max }}=27.5^{\circ}, \theta_{\text {min }}=5.1^{\circ}$

$h=-11 \rightarrow 11$

$k=-16 \rightarrow 17$

$l=-13 \rightarrow 13$

Primary atom site location: structure-invariant direct methods

Secondary atom site location: difference Fourier map

Hydrogen site location: mixed

$\mathrm{H}$ atoms treated by a mixture of independent and constrained refinement 
$w=1 /\left[\sigma^{2}\left(F_{0}^{2}\right)+(0.0345 P)^{2}\right]$

where $P=\left(F_{\mathrm{o}}^{2}+2 F_{\mathrm{c}}^{2}\right) / 3$

$(\Delta / \sigma)_{\max }<0.001$

$$
\Delta \rho_{\max }=0.17 \text { e } \AA^{-3}
$$$$
\Delta \rho_{\min }=-0.15 \text { e } \AA^{-3}
$$

\section{Special details}

Geometry. All e.s.d.'s (except the e.s.d. in the dihedral angle between two 1.s. planes) are estimated using the full covariance matrix. The cell e.s.d.'s are taken into account individually in the estimation of e.s.d.'s in distances, angles and torsion angles; correlations between e.s.d.'s in cell parameters are only used when they are defined by crystal symmetry. An approximate (isotropic) treatment of cell e.s.d.'s is used for estimating e.s.d.'s involving 1.s. planes.

Refinement. Refinement of $F^{2}$ against ALL reflections. The weighted $R$-factor $w R$ and goodness of fit $S$ are based on $F^{2}$, conventional $R$-factors $R$ are based on $F$, with $F$ set to zero for negative $F^{2}$. The threshold expression of $F^{2}>2 \sigma\left(F^{2}\right)$ is used only for calculating $R$-factors(gt) $e t c$. and is not relevant to the choice of reflections for refinement. $R$-factors based on $F^{2}$ are statistically about twice as large as those based on $F$, and $R$-factors based on ALL data will be even larger.

Fractional atomic coordinates and isotropic or equivalent isotropic displacement parameters $\left(\hat{A}^{2}\right)$

\begin{tabular}{|c|c|c|c|c|}
\hline & $x$ & $y$ & $z$ & $U_{\text {iso }} * / U_{\text {eq }}$ \\
\hline $\mathrm{O} 1$ & $0.4571(4)$ & $0.0371(2)$ & $0.1278(3)$ & $0.0510(9)$ \\
\hline $\mathrm{O} 2$ & $0.2161(4)$ & $0.4009(3)$ & 0.3492 (4) & $0.0591(10)$ \\
\hline $\mathrm{H} 2 \mathrm{O}$ & $0.284(6)$ & $0.426(4)$ & $0.307(5)$ & $0.061(15)^{*}$ \\
\hline $\mathrm{O} 3$ & 0.1944 (4) & $0.0677(2)$ & -0.0770 & $0.0660(10)$ \\
\hline $\mathrm{O} 4$ & $-0.0551(4)$ & $1.1537(3)$ & $0.4038(4)$ & $0.0686(11)$ \\
\hline $\mathrm{H} 4 \mathrm{O}$ & $-0.058(7)$ & $1.200(4)$ & $0.337(6)$ & $0.09(2)^{*}$ \\
\hline N1 & $0.4249(4)$ & $0.4745(3)$ & $0.2281(4)$ & $0.0448(10)$ \\
\hline $\mathrm{C} 1$ & $0.2616(5)$ & $0.8125(3)$ & $0.3304(4)$ & $0.0390(10)$ \\
\hline $\mathrm{C} 2$ & $0.1761(5)$ & 0.9019 (3) & $0.3470(4)$ & 0.0409 (11) \\
\hline $\mathrm{C} 3$ & $0.4907(6)$ & $0.2102(4)$ & $0.1807(5)$ & $0.0464(12)$ \\
\hline $\mathrm{H} 3$ & 0.5871 & 0.1998 & 0.2530 & $0.056^{*}$ \\
\hline $\mathrm{C} 4$ & $0.4297(5)$ & $0.3056(4)$ & $0.1500(5)$ & $0.0450(12)$ \\
\hline $\mathrm{C} 5$ & $0.4095(6)$ & $0.1326(3)$ & $0.1054(5)$ & $0.0450(12)$ \\
\hline C6 & $0.3543(6)$ & $0.6482(3)$ & $0.3954(5)$ & $0.0540(13)$ \\
\hline H6 & 0.3556 & 0.5936 & 0.4475 & $0.065^{*}$ \\
\hline $\mathrm{C} 7$ & $0.5309(5)$ & $0.5553(3)$ & $0.3000(5)$ & $0.0532(14)$ \\
\hline H7A & 0.6029 & 0.5719 & 0.2535 & $0.064^{*}$ \\
\hline H7B & 0.5969 & 0.5339 & 0.3900 & $0.064 *$ \\
\hline O5 & $-0.0663(6)$ & $0.3050(3)$ & $0.2485(5)$ & $0.0724(12)$ \\
\hline $\mathrm{H} 5 \mathrm{O}$ & $0.007(9)$ & $0.331(6)$ & $0.259(8)$ & $0.12(4)^{*}$ \\
\hline $\mathrm{C} 8$ & $0.5275(5)$ & $0.3884(3)$ & $0.2354(5)$ & 0.0519 (13) \\
\hline $\mathrm{H} 8 \mathrm{~A}$ & 0.5766 & 0.3671 & 0.3283 & $0.062 *$ \\
\hline H8B & 0.6136 & 0.4061 & 0.2045 & $0.062 *$ \\
\hline C9 & $0.2058(6)$ & $0.2421(3)$ & $-0.0333(5)$ & $0.0510(13)$ \\
\hline H9 & 0.1102 & 0.2529 & -0.1063 & $0.061^{*}$ \\
\hline $\mathrm{C} 10$ & $0.0835(6)$ & 0.9047 (4) & $0.4265(5)$ & $0.0532(13)$ \\
\hline H10 & 0.0709 & 0.8477 & 0.4688 & $0.064 *$ \\
\hline C11 & $0.2866(5)$ & $0.3213(3)$ & $0.0452(5)$ & $0.0456(12)$ \\
\hline $\mathrm{C} 12$ & $0.2151(6)$ & $0.4222(3)$ & $0.0158(5)$ & 0.0514 (13) \\
\hline $\mathrm{H} 12 \mathrm{~A}$ & 0.1242 & 0.4268 & 0.0452 & $0.062 *$ \\
\hline H12B & 0.1750 & 0.4335 & -0.0806 & $0.062 *$ \\
\hline C13 & $0.3421(6)$ & 0.8087 (3) & $0.2410(4)$ & $0.0491(12)$ \\
\hline
\end{tabular}




$\begin{array}{lllll}\text { H13 } & 0.3378 & 0.8621 & 0.1862 & 0.059^{*} \\ \text { C14 } & 0.1866(6) & 0.9887(3) & 0.2849(6) & 0.0602(15) \\ \text { H14 } & 0.2464 & 0.9906 & 0.2295 & 0.072^{*} \\ \text { C15 } & 0.3377(6) & 0.5000(3) & 0.0859(5) & 0.0527(14) \\ \text { H15A } & 0.4142 & 0.5069 & 0.0411 & 0.063^{*} \\ \text { H15B } & 0.2831 & 0.5619 & 0.0803 & 0.063^{*} \\ \text { C16 } & 0.2641(6) & 0.1490(4) & -0.0050(5) & 0.0469(11) \\ \text { C17 } & 0.2690(6) & 0.7294(3) & 0.4065(5) & 0.0542(14) \\ \text { H17 } & 0.2151 & 0.7285 & 0.4664 & 0.065^{*} \\ \text { C18 } & 0.4376(5) & 0.6453(3) & 0.3097(5) & 0.0459(12) \\ \text { C19 } & 0.0090(6) & 0.9887(4) & 0.4456(5) & 0.0578(14) \\ \text { H19 } & -0.0502 & 0.9877 & 0.5015 & 0.069^{*} \\ \text { C20 } & 0.0220(6) & 1.0731(4) & 0.3825(5) & 0.0522(13) \\ \text { C21 } & 0.4286(6) & 0.7268(3) & 0.2323(5) & 0.0538(13) \\ \text { H21 } & 0.4823 & 0.7270 & 0.1723 & 0.065^{*} \\ \text { C22 } & 0.1113(7) & 1.0729(4) & 0.3026(6) & 0.0646(15) \\ \text { H22 } & 0.1216 & 1.1300 & 0.2594 & 0.078^{*} \\ \text { C23 } & 0.0516(7) & 0.0832(5) & -0.1916(6) & 0.0864(19) \\ \text { H23A } & 0.0125 & 0.0219 & -0.2346 & 0.130^{*} \\ \text { H23B } & 0.0750 & 0.1257 & -0.2535 & 0.130^{*} \\ \text { H23C } & -0.0293 & 0.1126 & -0.1641 & 0.130^{*} \\ \text { C24 } & 0.3132(7) & 0.3394(4) & 0.4543(6) & 0.0847(19) \\ \text { H24A } & 0.2517 & 0.3176 & 0.5063 & 0.127^{*} \\ \text { H24B } & 0.4062 & 0.3749 & 0.5112 & 0.127^{*} \\ \text { H24C } & 0.3475 & 0.2841 & 0.4165 & 0.127^{*} \\ \text { C25 } & 0.6072(7) & 0.0187(4) & 0.2328(6) & 0.0707(17) \\ \text { H25A } & 0.6324 & -0.0494 & 0.2345 & 0.106^{*} \\ \text { H25B } & 0.6006 & 0.0371 & 0.3174 & 0.106^{*} \\ \text { H25C } & 0.6903 & 0.0561 & 0.2179 & 0.106^{*} \\ \text { C26 } & -0.1413(7) & 0.2993(5) & 0.1057(6) & 0.0846(19) \\ \text { H26A } & -0.1724 & 0.3634 & 0.0694 & 0.127^{*} \\ \text { H26B } & -0.2353 & 0.2585 & 0.0821 & 0.127^{*} \\ \text { H26C } & -0.0665 & 0.2721 & 0.0694 & 0.127^{*} \\ & & & & \end{array}$

Atomic displacement parameters $\left(\AA^{2}\right)$

\begin{tabular}{lllllll}
\hline & $U^{11}$ & $U^{22}$ & $U^{33}$ & $U^{12}$ & $U^{13}$ & $U^{23}$ \\
\hline O1 & $0.052(2)$ & $0.045(2)$ & $0.058(2)$ & $0.0013(16)$ & $0.0214(19)$ & $-0.0015(16)$ \\
O2 & $0.045(2)$ & $0.069(2)$ & $0.061(2)$ & $0.0081(19)$ & $0.0175(19)$ & $0.016(2)$ \\
O3 & $0.060(2)$ & $0.054(2)$ & $0.067(3)$ & $-0.0115(19)$ & $0.004(2)$ & $-0.016(2)$ \\
O4 & $0.079(3)$ & $0.058(2)$ & $0.085(3)$ & $0.008(2)$ & $0.049(2)$ & $-0.005(3)$ \\
N1 & $0.039(2)$ & $0.045(2)$ & $0.045(3)$ & $-0.0019(19)$ & $0.0086(18)$ & $-0.0088(19)$ \\
C1 & $0.037(3)$ & $0.045(3)$ & $0.034(3)$ & $-0.009(2)$ & $0.012(2)$ & $-0.009(2)$ \\
C2 & $0.039(3)$ & $0.047(3)$ & $0.035(3)$ & $-0.006(2)$ & $0.012(2)$ & $-0.004(2)$ \\
C3 & $0.042(3)$ & $0.058(3)$ & $0.039(3)$ & $0.004(3)$ & $0.014(2)$ & $-0.008(3)$ \\
C4 & $0.039(3)$ & $0.051(3)$ & $0.047(3)$ & $-0.002(3)$ & $0.019(2)$ & $-0.013(3)$ \\
C5 & $0.047(3)$ & $0.044(3)$ & $0.049(3)$ & $-0.001(2)$ & $0.023(3)$ & $-0.005(2)$ \\
C6 & $0.067(3)$ & $0.040(3)$ & $0.055(3)$ & $-0.004(3)$ & $0.023(3)$ & $0.003(3)$
\end{tabular}




$\begin{array}{lllllll}\text { C7 } & 0.049(3) & 0.048(3) & 0.062(3) & -0.004(3) & 0.020(3) & -0.015(3) \\ \text { O5 } & 0.071(3) & 0.067(3) & 0.075(3) & -0.010(3) & 0.022(2) & 0.001(2) \\ \text { C8 } & 0.042(3) & 0.047(3) & 0.065(4) & 0.003(2) & 0.017(3) & -0.013(3) \\ \text { C9 } & 0.044(3) & 0.054(3) & 0.047(3) & -0.002(3) & 0.007(2) & -0.007(3) \\ \text { C10 } & 0.061(3) & 0.056(3) & 0.051(3) & 0.002(3) & 0.030(3) & 0.011(3) \\ \text { C11 } & 0.043(3) & 0.045(3) & 0.047(3) & 0.002(2) & 0.014(3) & -0.009(2) \\ \text { C12 } & 0.047(3) & 0.050(3) & 0.049(3) & 0.004(3) & 0.009(2) & -0.003(3) \\ \text { C13 } & 0.058(3) & 0.044(3) & 0.048(3) & -0.003(3) & 0.021(3) & -0.001(2) \\ \text { C14 } & 0.070(4) & 0.049(3) & 0.081(4) & 0.005(3) & 0.052(3) & 0.004(3) \\ \text { C15 } & 0.054(3) & 0.052(3) & 0.053(4) & -0.004(3) & 0.020(3) & -0.004(3) \\ \text { C16 } & 0.047(3) & 0.048(3) & 0.047(3) & -0.007(3) & 0.018(3) & -0.010(3) \\ \text { C17 } & 0.070(3) & 0.047(3) & 0.057(4) & -0.007(3) & 0.036(3) & -0.004(3) \\ \text { C18 } & 0.043(3) & 0.045(3) & 0.046(3) & -0.004(2) & 0.012(2) & -0.009(3) \\ \text { C19 } & 0.062(3) & 0.062(4) & 0.063(4) & 0.005(3) & 0.039(3) & 0.001(3) \\ \text { C20 } & 0.049(3) & 0.049(3) & 0.063(3) & -0.003(3) & 0.026(3) & -0.007(3) \\ \text { C21 } & 0.065(3) & 0.047(3) & 0.057(4) & -0.008(3) & 0.032(3) & -0.008(3) \\ \text { C22 } & 0.074(4) & 0.052(3) & 0.089(4) & -0.002(3) & 0.055(4) & 0.007(3) \\ \text { C23 } & 0.074(4) & 0.078(4) & 0.079(4) & -0.017(3) & -0.005(4) & -0.024(4) \\ \text { C24 } & 0.074(4) & 0.100(5) & 0.067(4) & 0.020(4) & 0.010(3) & 0.028(4) \\ \text { C25 } & 0.069(4) & 0.063(4) & 0.068(4) & 0.015(3) & 0.012(3) & 0.002(3) \\ \text { C26 } & 0.078(4) & 0.106(5) & 0.073(5) & -0.022(4) & 0.031(4) & -0.018(4)\end{array}$

Geometric parameters $\left(\AA,{ }^{\circ}\right)$

\begin{tabular}{llll}
\hline $\mathrm{O} 1-\mathrm{C} 5$ & $1.371(5)$ & $\mathrm{C} 9-\mathrm{C} 11$ & $1.399(6)$ \\
$\mathrm{O} 1-\mathrm{C} 25$ & $1.415(6)$ & $\mathrm{C} 9-\mathrm{H} 9$ & 0.9300 \\
$\mathrm{O} 2-\mathrm{C} 24$ & $1.415(6)$ & $\mathrm{C} 10-\mathrm{C} 19$ & $1.380(7)$ \\
$\mathrm{O} 2-\mathrm{H} 2 \mathrm{O}$ & $0.94(5)$ & $\mathrm{C} 10-\mathrm{H} 10$ & 0.9300 \\
$\mathrm{O} 3-\mathrm{C} 16$ & $1.367(6)$ & $\mathrm{C} 11-\mathrm{C} 12$ & $1.508(6)$ \\
$\mathrm{O} 3-\mathrm{C} 23$ & $1.419(6)$ & $\mathrm{C} 12-\mathrm{C} 15$ & $1.513(6)$ \\
$\mathrm{O} 4-\mathrm{C} 20$ & $1.364(5)$ & $\mathrm{C} 12-\mathrm{H} 12 \mathrm{~A}$ & 0.9700 \\
$\mathrm{O} 4-\mathrm{H} 4 \mathrm{O}$ & $0.95(6)$ & $\mathrm{C} 12-\mathrm{H} 12 \mathrm{~B}$ & 0.9700 \\
$\mathrm{~N} 1-\mathrm{C} 15$ & $1.471(6)$ & $\mathrm{C} 13-\mathrm{C} 21$ & $1.383(6)$ \\
$\mathrm{N} 1-\mathrm{C} 7$ & $1.475(5)$ & $\mathrm{C} 13-\mathrm{H} 13$ & 0.9300 \\
$\mathrm{~N} 1-\mathrm{C} 8$ & $1.477(5)$ & $\mathrm{C} 14-\mathrm{C} 22$ & $1.382(7)$ \\
$\mathrm{C} 1-\mathrm{C} 17$ & $1.388(6)$ & $\mathrm{C} 14-\mathrm{H} 14$ & 0.9300 \\
$\mathrm{C} 1-\mathrm{C} 13$ & $1.389(6)$ & $\mathrm{C} 15-\mathrm{H} 15 \mathrm{~A}$ & 0.9700 \\
$\mathrm{C} 1-\mathrm{C} 2$ & $1.488(6)$ & $\mathrm{C} 15-\mathrm{H} 15 \mathrm{~B}$ & 0.9700 \\
$\mathrm{C} 2-\mathrm{C} 14$ & $\mathrm{C} 17-\mathrm{H} 17$ & 0.9300 \\
$\mathrm{C} 2-\mathrm{C} 10$ & $1.383(6)$ & $\mathrm{C} 18-\mathrm{C} 21$ & $1.376(6)$ \\
$\mathrm{C} 3-\mathrm{C} 5$ & $1.384(6)$ & $\mathrm{C} 19-\mathrm{C} 20$ & $1.366(6)$ \\
$\mathrm{C} 3-\mathrm{C} 4$ & $1.369(6)$ & $\mathrm{C} 19-\mathrm{H} 19$ & 0.9300 \\
$\mathrm{C} 3-\mathrm{H} 3$ & $1.409(6)$ & $\mathrm{C} 20-\mathrm{C} 22$ & $1.364(6)$ \\
$\mathrm{C} 4-\mathrm{C} 11$ & $\mathrm{C} 21-\mathrm{H} 21$ & 0.9300 \\
$\mathrm{C} 4-\mathrm{C} 8$ & 1.9300 & $\mathrm{C} 22-\mathrm{H} 22$ & 0.9300 \\
$\mathrm{C} 5-\mathrm{C} 16$ & $1.567(6)$ & $\mathrm{C} 23-\mathrm{H} 23 \mathrm{~A}$ & 0.9600 \\
$\mathrm{C} 6-\mathrm{C} 18$ & $1.411(6)$ & $\mathrm{C} 23-\mathrm{H} 23 \mathrm{~B}$ & 0.9600 \\
$\mathrm{C} 6-\mathrm{C} 17$ & $1.373(6)$ & $\mathrm{C} 23-\mathrm{H} 23 \mathrm{C}$ & 0.9600 \\
& $1.378(6)$ & &
\end{tabular}




\begin{tabular}{|c|c|c|c|}
\hline $\mathrm{C} 6-\mathrm{H} 6$ & 0.9300 & $\mathrm{C} 24-\mathrm{H} 24 \mathrm{~A}$ & 0.9600 \\
\hline $\mathrm{C} 7-\mathrm{C} 18$ & $1.511(6)$ & $\mathrm{C} 24-\mathrm{H} 24 \mathrm{~B}$ & 0.9600 \\
\hline $\mathrm{C} 7-\mathrm{H} 7 \mathrm{~A}$ & 0.9700 & $\mathrm{C} 24-\mathrm{H} 24 \mathrm{C}$ & 0.9600 \\
\hline C7-H7B & 0.9700 & $\mathrm{C} 25-\mathrm{H} 25 \mathrm{~A}$ & 0.9600 \\
\hline $\mathrm{O} 5-\mathrm{C} 26$ & $1.424(7)$ & $\mathrm{C} 25-\mathrm{H} 25 \mathrm{~B}$ & 0.9600 \\
\hline $\mathrm{O} 5-\mathrm{H} 5 \mathrm{O}$ & $0.71(8)$ & $\mathrm{C} 25-\mathrm{H} 25 \mathrm{C}$ & 0.9600 \\
\hline $\mathrm{C} 8-\mathrm{H} 8 \mathrm{~A}$ & 0.9700 & $\mathrm{C} 26-\mathrm{H} 26 \mathrm{~A}$ & 0.9600 \\
\hline $\mathrm{C} 8-\mathrm{H} 8 \mathrm{~B}$ & 0.9700 & $\mathrm{C} 26-\mathrm{H} 26 \mathrm{~B}$ & 0.9600 \\
\hline $\mathrm{C} 9-\mathrm{C} 16$ & $1.370(7)$ & $\mathrm{C} 26-\mathrm{H} 26 \mathrm{C}$ & 0.9600 \\
\hline $\mathrm{C} 5-\mathrm{O} 1-\mathrm{C} 25$ & $116.7(4)$ & $\mathrm{C} 1-\mathrm{C} 13-\mathrm{H} 13$ & 119.4 \\
\hline $\mathrm{C} 24-\mathrm{O} 2-\mathrm{H} 2 \mathrm{O}$ & $107(3)$ & $\mathrm{C} 22-\mathrm{C} 14-\mathrm{C} 2$ & $122.1(5)$ \\
\hline $\mathrm{C} 16-\mathrm{O} 3-\mathrm{C} 23$ & $116.1(4)$ & $\mathrm{C} 22-\mathrm{C} 14-\mathrm{H} 14$ & 118.9 \\
\hline $\mathrm{C} 20-\mathrm{O} 4-\mathrm{H} 4 \mathrm{O}$ & $107(3)$ & $\mathrm{C} 2-\mathrm{C} 14-\mathrm{H} 14$ & 118.9 \\
\hline $\mathrm{C} 15-\mathrm{N} 1-\mathrm{C} 7$ & $110.6(4)$ & $\mathrm{N} 1-\mathrm{C} 15-\mathrm{C} 12$ & $110.7(4)$ \\
\hline $\mathrm{C} 15-\mathrm{N} 1-\mathrm{C} 8$ & $109.0(4)$ & $\mathrm{N} 1-\mathrm{C} 15-\mathrm{H} 15 \mathrm{~A}$ & 109.5 \\
\hline $\mathrm{C} 7-\mathrm{N} 1-\mathrm{C} 8$ & $108.0(3)$ & $\mathrm{C} 12-\mathrm{C} 15-\mathrm{H} 15 \mathrm{~A}$ & 109.5 \\
\hline $\mathrm{C} 17-\mathrm{C} 1-\mathrm{C} 13$ & $116.5(4)$ & $\mathrm{N} 1-\mathrm{C} 15-\mathrm{H} 15 \mathrm{~B}$ & 109.5 \\
\hline $\mathrm{C} 17-\mathrm{C} 1-\mathrm{C} 2$ & $121.6(4)$ & $\mathrm{C} 12-\mathrm{C} 15-\mathrm{H} 15 \mathrm{~B}$ & 109.5 \\
\hline $\mathrm{C} 13-\mathrm{C} 1-\mathrm{C} 2$ & $121.9(4)$ & $\mathrm{H} 15 \mathrm{~A}-\mathrm{C} 15-\mathrm{H} 15 \mathrm{~B}$ & 108.1 \\
\hline $\mathrm{C} 14-\mathrm{C} 2-\mathrm{C} 10$ & $115.5(4)$ & $\mathrm{O} 3-\mathrm{C} 16-\mathrm{C} 9$ & $125.5(4)$ \\
\hline $\mathrm{C} 14-\mathrm{C} 2-\mathrm{C} 1$ & $121.6(4)$ & $\mathrm{O} 3-\mathrm{C} 16-\mathrm{C} 5$ & $115.2(5)$ \\
\hline $\mathrm{C} 10-\mathrm{C} 2-\mathrm{C} 1$ & $122.8(4)$ & $\mathrm{C} 9-\mathrm{C} 16-\mathrm{C} 5$ & $119.3(5)$ \\
\hline $\mathrm{C} 5-\mathrm{C} 3-\mathrm{C} 4$ & $120.7(4)$ & $\mathrm{C} 6-\mathrm{C} 17-\mathrm{C} 1$ & $121.5(5)$ \\
\hline $\mathrm{C} 5-\mathrm{C} 3-\mathrm{H} 3$ & 119.6 & $\mathrm{C} 6-\mathrm{C} 17-\mathrm{H} 17$ & 119.2 \\
\hline $\mathrm{C} 4-\mathrm{C} 3-\mathrm{H} 3$ & 119.6 & $\mathrm{C} 1-\mathrm{C} 17-\mathrm{H} 17$ & 119.2 \\
\hline $\mathrm{C} 11-\mathrm{C} 4-\mathrm{C} 3$ & $120.0(4)$ & $\mathrm{C} 6-\mathrm{C} 18-\mathrm{C} 21$ & $116.7(5)$ \\
\hline $\mathrm{C} 11-\mathrm{C} 4-\mathrm{C} 8$ & $122.0(4)$ & $\mathrm{C} 6-\mathrm{C} 18-\mathrm{C} 7$ & $120.9(5)$ \\
\hline $\mathrm{C} 3-\mathrm{C} 4-\mathrm{C} 8$ & $118.0(4)$ & $\mathrm{C} 21-\mathrm{C} 18-\mathrm{C} 7$ & $122.3(5)$ \\
\hline $\mathrm{C} 3-\mathrm{C} 5-\mathrm{O} 1$ & $125.4(5)$ & $\mathrm{C} 20-\mathrm{C} 19-\mathrm{C} 10$ & $120.2(5)$ \\
\hline $\mathrm{C} 3-\mathrm{C} 5-\mathrm{C} 16$ & $119.3(5)$ & $\mathrm{C} 20-\mathrm{C} 19-\mathrm{H} 19$ & 119.9 \\
\hline $\mathrm{O} 1-\mathrm{C} 5-\mathrm{C} 16$ & $115.3(4)$ & $\mathrm{C} 10-\mathrm{C} 19-\mathrm{H} 19$ & 119.9 \\
\hline $\mathrm{C} 18-\mathrm{C} 6-\mathrm{C} 17$ & $122.0(5)$ & $\mathrm{C} 22-\mathrm{C} 20-\mathrm{O} 4$ & $123.4(5)$ \\
\hline $\mathrm{C} 18-\mathrm{C} 6-\mathrm{H} 6$ & 119.0 & $\mathrm{C} 22-\mathrm{C} 20-\mathrm{C} 19$ & $118.8(5)$ \\
\hline $\mathrm{C} 17-\mathrm{C} 6-\mathrm{H} 6$ & 119.0 & $\mathrm{O} 4-\mathrm{C} 20-\mathrm{C} 19$ & $117.8(5)$ \\
\hline $\mathrm{N} 1-\mathrm{C} 7-\mathrm{C} 18$ & $112.8(4)$ & $\mathrm{C} 18-\mathrm{C} 21-\mathrm{C} 13$ & $122.1(5)$ \\
\hline $\mathrm{N} 1-\mathrm{C} 7-\mathrm{H} 7 \mathrm{~A}$ & 109.0 & $\mathrm{C} 18-\mathrm{C} 21-\mathrm{H} 21$ & 119.0 \\
\hline $\mathrm{C} 18-\mathrm{C} 7-\mathrm{H} 7 \mathrm{~A}$ & 109.0 & $\mathrm{C} 13-\mathrm{C} 21-\mathrm{H} 21$ & 119.0 \\
\hline $\mathrm{N} 1-\mathrm{C} 7-\mathrm{H} 7 \mathrm{~B}$ & 109.0 & $\mathrm{C} 20-\mathrm{C} 22-\mathrm{C} 14$ & $120.7(5)$ \\
\hline $\mathrm{C} 18-\mathrm{C} 7-\mathrm{H} 7 \mathrm{~B}$ & 109.0 & $\mathrm{C} 20-\mathrm{C} 22-\mathrm{H} 22$ & 119.6 \\
\hline $\mathrm{H} 7 \mathrm{~A}-\mathrm{C} 7-\mathrm{H} 7 \mathrm{~B}$ & 107.8 & $\mathrm{C} 14-\mathrm{C} 22-\mathrm{H} 22$ & 119.6 \\
\hline $\mathrm{C} 26-\mathrm{O} 5-\mathrm{H} 5 \mathrm{O}$ & $104(7)$ & $\mathrm{O} 3-\mathrm{C} 23-\mathrm{H} 23 \mathrm{~A}$ & 109.5 \\
\hline $\mathrm{N} 1-\mathrm{C} 8-\mathrm{C} 4$ & $111.2(4)$ & $\mathrm{O} 3-\mathrm{C} 23-\mathrm{H} 23 \mathrm{~B}$ & 109.5 \\
\hline $\mathrm{N} 1-\mathrm{C} 8-\mathrm{H} 8 \mathrm{~A}$ & 109.4 & $\mathrm{H} 23 \mathrm{~A}-\mathrm{C} 23-\mathrm{H} 23 \mathrm{~B}$ & 109.5 \\
\hline $\mathrm{C} 4-\mathrm{C} 8-\mathrm{H} 8 \mathrm{~A}$ & 109.4 & $\mathrm{O} 3-\mathrm{C} 23-\mathrm{H} 23 \mathrm{C}$ & 109.5 \\
\hline $\mathrm{N} 1-\mathrm{C} 8-\mathrm{H} 8 \mathrm{~B}$ & 109.4 & $\mathrm{H} 23 \mathrm{~A}-\mathrm{C} 23-\mathrm{H} 23 \mathrm{C}$ & 109.5 \\
\hline $\mathrm{C} 4-\mathrm{C} 8-\mathrm{H} 8 \mathrm{~B}$ & 109.4 & $\mathrm{H} 23 \mathrm{~B}-\mathrm{C} 23-\mathrm{H} 23 \mathrm{C}$ & 109.5 \\
\hline $\mathrm{H} 8 \mathrm{~A}-\mathrm{C} 8-\mathrm{H} 8 \mathrm{~B}$ & 108.0 & $\mathrm{O} 2-\mathrm{C} 24-\mathrm{H} 24 \mathrm{~A}$ & 109.5 \\
\hline
\end{tabular}




\begin{tabular}{|c|c|}
\hline $\mathrm{C} 16-\mathrm{C} 9-\mathrm{C} 11$ & $121.5(5)$ \\
\hline $\mathrm{C} 16-\mathrm{C} 9-\mathrm{H} 9$ & 119.3 \\
\hline $\mathrm{C} 11-\mathrm{C} 9-\mathrm{H} 9$ & 119.3 \\
\hline $\mathrm{C} 19-\mathrm{C} 10-\mathrm{C} 2$ & $122.7(5)$ \\
\hline $\mathrm{C} 19-\mathrm{C} 10-\mathrm{H} 10$ & 118.7 \\
\hline $\mathrm{C} 2-\mathrm{C} 10-\mathrm{H} 10$ & 118.7 \\
\hline $\mathrm{C} 4-\mathrm{C} 11-\mathrm{C} 9$ & $119.1(4)$ \\
\hline $\mathrm{C} 4-\mathrm{C} 11-\mathrm{C} 12$ & $120.6(4)$ \\
\hline $\mathrm{C} 9-\mathrm{C} 11-\mathrm{C} 12$ & $120.2(4)$ \\
\hline $\mathrm{C} 11-\mathrm{C} 12-\mathrm{C} 15$ & $111.8(4)$ \\
\hline $\mathrm{C} 11-\mathrm{C} 12-\mathrm{H} 12 \mathrm{~A}$ & 109.2 \\
\hline $\mathrm{C} 15-\mathrm{C} 12-\mathrm{H} 12 \mathrm{~A}$ & 109.2 \\
\hline $\mathrm{C} 11-\mathrm{C} 12-\mathrm{H} 12 \mathrm{~B}$ & 109.2 \\
\hline $\mathrm{C} 15-\mathrm{C} 12-\mathrm{H} 12 \mathrm{~B}$ & 109.2 \\
\hline $\mathrm{H} 12 \mathrm{~A}-\mathrm{C} 12-\mathrm{H} 12 \mathrm{~B}$ & 107.9 \\
\hline $\mathrm{C} 21-\mathrm{C} 13-\mathrm{C} 1$ & $121.1(4)$ \\
\hline $\mathrm{C} 21-\mathrm{C} 13-\mathrm{H} 13$ & 119.4 \\
\hline $\mathrm{C} 17-\mathrm{C} 1-\mathrm{C} 2-\mathrm{C} 14$ & $171.2(5)$ \\
\hline $\mathrm{C} 13-\mathrm{C} 1-\mathrm{C} 2-\mathrm{C} 14$ & $-6.9(6)$ \\
\hline $\mathrm{C} 17-\mathrm{C} 1-\mathrm{C} 2-\mathrm{C} 10$ & $-7.7(7)$ \\
\hline $\mathrm{C} 13-\mathrm{C} 1-\mathrm{C} 2-\mathrm{C} 10$ & $174.2(5)$ \\
\hline $\mathrm{C} 5-\mathrm{C} 3-\mathrm{C} 4-\mathrm{C} 11$ & $1.1(6)$ \\
\hline $\mathrm{C} 5-\mathrm{C} 3-\mathrm{C} 4-\mathrm{C} 8$ & $-178.6(4)$ \\
\hline $\mathrm{C} 4-\mathrm{C} 3-\mathrm{C} 5-\mathrm{O} 1$ & $-179.1(4)$ \\
\hline $\mathrm{C} 4-\mathrm{C} 3-\mathrm{C} 5-\mathrm{C} 16$ & $0.7(6)$ \\
\hline $\mathrm{C} 25-\mathrm{O} 1-\mathrm{C} 5-\mathrm{C} 3$ & $-3.8(6)$ \\
\hline $\mathrm{C} 25-\mathrm{O} 1-\mathrm{C} 5-\mathrm{C} 16$ & $176.4(4)$ \\
\hline $\mathrm{C} 15-\mathrm{N} 1-\mathrm{C} 7-\mathrm{C} 18$ & $65.8(5)$ \\
\hline $\mathrm{C} 8-\mathrm{N} 1-\mathrm{C} 7-\mathrm{C} 18$ & $-175.0(4)$ \\
\hline $\mathrm{C} 15-\mathrm{N} 1-\mathrm{C} 8-\mathrm{C} 4$ & $-53.1(5)$ \\
\hline $\mathrm{C} 7-\mathrm{N} 1-\mathrm{C} 8-\mathrm{C} 4$ & $-173.4(4)$ \\
\hline $\mathrm{C} 11-\mathrm{C} 4-\mathrm{C} 8-\mathrm{N} 1$ & $22.3(6)$ \\
\hline $\mathrm{C} 3-\mathrm{C} 4-\mathrm{C} 8-\mathrm{N} 1$ & $-158.0(4)$ \\
\hline $\mathrm{C} 14-\mathrm{C} 2-\mathrm{C} 10-\mathrm{C} 19$ & $-1.4(7)$ \\
\hline $\mathrm{C} 1-\mathrm{C} 2-\mathrm{C} 10-\mathrm{C} 19$ & $177.6(5)$ \\
\hline $\mathrm{C} 3-\mathrm{C} 4-\mathrm{C} 11-\mathrm{C} 9$ & $-2.4(6)$ \\
\hline $\mathrm{C} 8-\mathrm{C} 4-\mathrm{C} 11-\mathrm{C} 9$ & $177.3(4)$ \\
\hline $\mathrm{C} 3-\mathrm{C} 4-\mathrm{C} 11-\mathrm{C} 12$ & $176.6(4)$ \\
\hline $\mathrm{C} 8-\mathrm{C} 4-\mathrm{C} 11-\mathrm{C} 12$ & $-3.8(6)$ \\
\hline $\mathrm{C} 16-\mathrm{C} 9-\mathrm{C} 11-\mathrm{C} 4$ & $2.0(7)$ \\
\hline $\mathrm{C} 16-\mathrm{C} 9-\mathrm{C} 11-\mathrm{C} 12$ & $-176.9(4)$ \\
\hline $\mathrm{C} 4-\mathrm{C} 11-\mathrm{C} 12-\mathrm{C} 15$ & $15.9(6)$ \\
\hline $\mathrm{C} 9-\mathrm{C} 11-\mathrm{C} 12-\mathrm{C} 15$ & $-165.2(4)$ \\
\hline $\mathrm{C} 17-\mathrm{C} 1-\mathrm{C} 13-\mathrm{C} 21$ & $-1.8(7)$ \\
\hline $\mathrm{C} 2-\mathrm{C} 1-\mathrm{C} 13-\mathrm{C} 21$ & $176.3(4)$ \\
\hline $\mathrm{C} 10-\mathrm{C} 2-\mathrm{C} 14-\mathrm{C} 22$ & $0.8(8)$ \\
\hline
\end{tabular}

$\begin{array}{ll}\mathrm{O} 2-\mathrm{C} 24-\mathrm{H} 24 \mathrm{~B} & 109.5 \\ \mathrm{H} 24 \mathrm{~A}-\mathrm{C} 24-\mathrm{H} 24 \mathrm{~B} & 109.5 \\ \mathrm{O} 2-\mathrm{C} 24-\mathrm{H} 24 \mathrm{C} & 109.5 \\ \mathrm{H} 24 \mathrm{~A}-\mathrm{C} 24-\mathrm{H} 24 \mathrm{C} & 109.5 \\ \mathrm{H} 24 \mathrm{~B}-\mathrm{C} 24-\mathrm{H} 24 \mathrm{C} & 109.5 \\ \mathrm{O} 1-\mathrm{C} 25-\mathrm{H} 25 \mathrm{~A} & 109.5 \\ \mathrm{O} 1-\mathrm{C} 25-\mathrm{H} 25 \mathrm{~B} & 109.5 \\ \mathrm{H} 25 \mathrm{~A}-\mathrm{C} 25-\mathrm{H} 25 \mathrm{~B} & 109.5 \\ \mathrm{O} 1-\mathrm{C} 25-\mathrm{H} 25 \mathrm{C} & 109.5 \\ \mathrm{H} 25 \mathrm{~A}-\mathrm{C} 25-\mathrm{H} 25 \mathrm{C} & 109.5 \\ \mathrm{H} 25 \mathrm{~B}-\mathrm{C} 25-\mathrm{H} 25 \mathrm{C} & 109.5 \\ \mathrm{O} 5-\mathrm{C} 26-\mathrm{H} 26 \mathrm{~A} & 109.5 \\ \mathrm{O} 5-\mathrm{C} 26-\mathrm{H} 26 \mathrm{~B} & 109.5 \\ \mathrm{H} 26 \mathrm{~A}-\mathrm{C} 26-\mathrm{H} 26 \mathrm{~B} & 109.5 \\ \mathrm{O} 5-\mathrm{C} 26-\mathrm{H} 26 \mathrm{C} & 109.5 \\ \mathrm{H} 26 \mathrm{~A}-\mathrm{C} 26-\mathrm{H} 26 \mathrm{C} & 109.5 \\ \mathrm{H} 26 \mathrm{~B}-\mathrm{C} 26-\mathrm{H} 26 \mathrm{C} & 109.5\end{array}$

$\mathrm{C} 1-\mathrm{C} 2-\mathrm{C} 14-\mathrm{C} 22 \quad-178.2(5)$

$\mathrm{C} 7-\mathrm{N} 1-\mathrm{C} 15-\mathrm{C} 12 \quad-173.6(4)$

$\mathrm{C} 8-\mathrm{N} 1-\mathrm{C} 15-\mathrm{C} 12$

$\mathrm{C} 11-\mathrm{C} 12-\mathrm{C} 15-\mathrm{N} 1 \quad-47.5(5)$

$\mathrm{C} 23-\mathrm{O} 3-\mathrm{C} 16-\mathrm{C} 9 \quad 1.4(7)$

$\mathrm{C} 23-\mathrm{O} 3-\mathrm{C} 16-\mathrm{C} 5 \quad-177.6(4)$

$\mathrm{C} 11-\mathrm{C} 9-\mathrm{C} 16-\mathrm{O} 3 \quad-179.2(4)$

$\mathrm{C} 11-\mathrm{C} 9-\mathrm{C} 16-\mathrm{C} 5 \quad-0.3(7)$

$\mathrm{C} 3-\mathrm{C} 5-\mathrm{C} 16-\mathrm{O} 3 \quad 177.9$ (4)

$\mathrm{O} 1-\mathrm{C} 5-\mathrm{C} 16-\mathrm{O} 3 \quad-2.3(5)$

$\mathrm{C} 3-\mathrm{C} 5-\mathrm{C} 16-\mathrm{C} 9 \quad-1.1(6)$

$\mathrm{O} 1-\mathrm{C} 5-\mathrm{C} 16-\mathrm{C} 9 \quad 178.7$ (4)

$\mathrm{C} 18-\mathrm{C} 6-\mathrm{C} 17-\mathrm{C} 1 \quad 0.6(7)$

$\mathrm{C} 13-\mathrm{C} 1-\mathrm{C} 17-\mathrm{C} 6 \quad 1.1(7)$

$\mathrm{C} 2-\mathrm{C} 1-\mathrm{C} 17-\mathrm{C} 6 \quad-177.0$ (4)

$\mathrm{C} 17-\mathrm{C} 6-\mathrm{C} 18-\mathrm{C} 21 \quad-1.6(7)$

$\mathrm{C} 17-\mathrm{C} 6-\mathrm{C} 18-\mathrm{C} 7 \quad 179.7$ (4)

$\mathrm{N} 1-\mathrm{C} 7-\mathrm{C} 18-\mathrm{C} 6 \quad 73.2(6)$

$\mathrm{N} 1-\mathrm{C} 7-\mathrm{C} 18-\mathrm{C} 21 \quad-105.4(5)$

$\mathrm{C} 2-\mathrm{C} 10-\mathrm{C} 19-\mathrm{C} 20 \quad 1.5(8)$

$\mathrm{C} 10-\mathrm{C} 19-\mathrm{C} 20-\mathrm{C} 22 \quad-0.9(8)$

$\mathrm{C} 10-\mathrm{C} 19-\mathrm{C} 20-\mathrm{O} 4 \quad 179.4(5)$

$\mathrm{C} 6-\mathrm{C} 18-\mathrm{C} 21-\mathrm{C} 13 \quad 0.9(7)$

$\mathrm{C} 7-\mathrm{C} 18-\mathrm{C} 21-\mathrm{C} 13 \quad 179.6(4)$

$\mathrm{C} 1-\mathrm{C} 13-\mathrm{C} 21-\mathrm{C} 18 \quad 0.8(8)$

$\mathrm{O} 4-\mathrm{C} 20-\mathrm{C} 22-\mathrm{C} 14 \quad 180.0(5)$

$\mathrm{C} 19-\mathrm{C} 20-\mathrm{C} 22-\mathrm{C} 14 \quad 0.4(8)$

$\mathrm{C} 2-\mathrm{C} 14-\mathrm{C} 22-\mathrm{C} 20 \quad-0.3(9)$ 


\section{supporting information}

Hydrogen-bond geometry $\left(\AA,{ }^{\circ}\right)$

\begin{tabular}{lllll}
\hline$D-\mathrm{H} \cdots A$ & $D-\mathrm{H}$ & $\mathrm{H} \cdots A$ & $D \cdots A$ & $D-\mathrm{H} \cdots A$ \\
\hline $\mathrm{O} 2-\mathrm{H} 2 O \cdots \mathrm{N} 1$ & $0.94(5)$ & $1.87(5)$ & $2.812(5)$ & $178(4)$ \\
$\mathrm{O} 4-\mathrm{H} 4 O \cdots \mathrm{O} 5^{\mathrm{i}}$ & $0.95(6)$ & $1.71(6)$ & $2.636(6)$ & $165(5)$ \\
$\mathrm{O} 5-\mathrm{H} 5 O \cdots \mathrm{O} 2$ & $0.71(8)$ & $2.00(8)$ & $2.684(6)$ & $162(9)$ \\
$\mathrm{C} 15-\mathrm{H} 15 A \cdots \mathrm{O} 1^{\mathrm{ii}}$ & 0.97 & 2.50 & $3.445(6)$ & 164 \\
$\mathrm{C} 23-\mathrm{H} 23 A \cdots \mathrm{O} 2^{\mathrm{iii}}$ & 0.96 & 2.56 & $3.437(6)$ & 152 \\
\hline
\end{tabular}

Symmetry codes: (i) $x, y+1, z$; (ii) $-x+1, y+1 / 2,-z$; (iii) $-x, y-1 / 2,-z$. 